\title{
A new method for gravity correction of dynamometer data and determining passive elastic moments at the joint
}

\section{Citation}

Anderson, Dennis E., Maury A. Nussbaum, and Michael L. Madigan. 2010. "A New Method for Gravity Correction of Dynamometer Data and Determining Passive Elastic Moments at the Joint." Journal of Biomechanics 43 (6) (April): 1220-1223. doi:10.1016/j.jbiomech.2009.11.036.

\section{Published Version}

doi:https://dx.doi.org/10.1016\%2Fj.jbiomech.2009.11.036

\section{Permanent link}

http://nrs.harvard.edu/urn-3:HUL.InstRepos:37133659

\section{Terms of Use}

This article was downloaded from Harvard University's DASH repository, and is made available under the terms and conditions applicable to Other Posted Material, as set forth at http:// nrs.harvard.edu/urn-3:HUL.InstRepos:dash.current.terms-of-use\#LAA

\section{Share Your Story}

The Harvard community has made this article openly available.

Please share how this access benefits you. Submit a story.

\section{Accessibility}




\title{
A new method for gravity correction of dynamometer data and determining passive elastic moments at the joint
}

\author{
Dennis E. Anderson ${ }^{1}$, Maury A. Nussbaum ${ }^{2,3,4}$, and Michael L. Madigan 1,2,3 \\ ${ }^{1}$ Department of Engineering Science and Mechanics \\ ${ }^{2}$ Virginia Tech-Wake Forest School of Biomedical Engineering and Sciences \\ ${ }^{3}$ Virginia Tech Center for Gerontology \\ ${ }^{4}$ Department of Industrial and Systems Engineering, Virginia Polytechnic Institute and State \\ University, Blacksburg
}

\begin{abstract}
Moments measured by a dynamometer in biomechanics testing often include the gravitational moment and the passive elastic moment in addition to the moment caused by muscle contraction. Gravitational moments result from the weight of body segments and dynamometer attachment, whereas passive elastic moments are caused by the passive elastic deformation of tissues crossing the joint being assessed. Gravitational moments are a major potential source of error in dynamometer measurements and must be corrected for, a procedure often called gravity correction. While several approaches to gravity correction have been presented in the literature, they generally assume that the gravitational moment can be adequately modeled as a simple sine or cosine function. With this approach, a single passive data point may be used to specify the model, assuming that passive elastic moments are negligible at that point. A new method is presented here for the gravity correction of dynamometer data. Gravitational moment is represented using a generalized sinusoid, which is fit to passive data obtained over the entire joint range of motion. The model also explicitly accounts for the presence of passive elastic moments. The model was tested for cases of hip flexion-extension, knee flexion-extension, and ankle plantar flexion-dorsiflexion, and provided good fits in all cases.
\end{abstract}

\section{Keywords}

isokinetic dynamometer; joint moment; gravity correction; gravitational moment; passive elastic moment

\section{Introduction}

Dynamometers are widely used in biomechanics research to measure the resultant moment at a joint due to muscular contraction. However, the moment measured by a dynamometer may

(C) 2009 Elsevier Ltd. All rights reserved.

Please address all correspondence to: Michael L. Madigan, Department of Engineering Science and Mechanics (MC 0219), Virginia Polytechnic Institute and State University, Blacksburg, VA 24061, Phone: (540) 231-1215, Fax: (540) 231-4574, mlm@ vt.edu.

Publisher's Disclaimer: This is a PDF file of an unedited manuscript that has been accepted for publication. As a service to our customers we are providing this early version of the manuscript. The manuscript will undergo copyediting, typesetting, and review of the resulting proof before it is published in its final citable form. Please note that during the production process errors may be discovered which could affect the content, and all legal disclaimers that apply to the journal pertain.

Conflict of interest statement

There is no conflict of interest to report. 
include not only the moment due to muscular contraction, but also gravitational moment and passive elastic moment at the joint. Gravitational moments are moments due to the weight of the limb and dynamometer attachment. As such, they are a major source of potential error in dynamometer measurements and must be corrected for (Herzog 1988). Passive elastic moments are caused primarily by the passive elastic deformation of tissues crossing the joint, including muscles, tendons and ligaments (Yoon and Mansour, 1982). To isolate the moment due to muscular contraction, gravitational and passive elastic moments must be determined and subtracted from dynamometer data.

A variety of approaches have been described in the literature for determining gravitational moments to subtract from measured dynamometer moments, a procedure often referred to as gravity correction. Several of these share the underlying assumption that the gravitational moment may be modeled by a simple sine or cosine function, but differ in the method used to parameterize the model (e.g. Ford et al. 1994; Kellis and Baltzopoulos 1996; Nelson and Duncan 1983; Westing and Seger 1989; Winter et al. 1981). Modeling gravitational moment as a simple sine or cosine function allows the model to be parameterized using a measurement of passive moment at a single angle by the dynamometer, but the resulting functions can vary greatly depending on the angle chosen (Keating and Matyas 1996). There are two main reasons for this. First, this approach assumes that zero gravitational moment occurs at a known angle, such as when the dynamometer arm is perpendicular to the ground. This assumption is not necessarily valid because the center of mass of the limb-dynamometer arm system not necessarily known, and is not necessarily aligned with the dynamometer attachment (Keating and Matyas 1996). Second, this approach assumes that there is no passive elastic moment present at the chosen angle (Kellis and Baltzopoulos 1996), but passive elastic moment can affect measured gravitational moment if care is not taken to minimize it (Ford et al. 1994; Gajdosik et al. 2001). The passive elastic moment may be non-negligible through a considerable portion of the range of motion (ROM), and the position of one joint can affect the passive elastic moment at another (Riener and Edrich 1999; Yoon and Mansour 1982), making the selection of an angle with minimal passive elastic moment difficult.

This paper presents a method for modeling gravitational and passive elastic moments based on dynamometer data collected during passive isokinetic motion through the entire joint ROM. Gravitational moment is modeled using a generalized sinusoid, allowing zero gravitational moment to occur at any dynamometer or joint angle. Moreover, this method accounts for passive elastic moments and models them separately from the gravitational moment.

\section{Methods}

Passive moment data collected from twenty healthy, active older adults (ages $55-73$ years, ten males, ten females) was used from a strength testing study that has been reported previously (Anderson et al. 2007). The study was approved by the Virginia Tech Institutional Review Board, and all participants gave written informed consent prior to participation. Testing was performed using a Biodex System 3 dynamometer (Biodex Medical Systems, Inc., Shirley, New York, USA). The dynamometer moved through two full cycles of joint motion in passive mode at $5 \%$ while participants remained relaxed. Moment, angle, and angular velocity were sampled at $200 \mathrm{~Hz}$ for hip flexion-extension, knee flexion-extension, and ankle plantar flexiondorsiflexion tests.

As noted, gravitational moment $(M G)$ is usually modeled using sine or cosine functions (Herzog 1988; Nelson and Duncan 1983; Winter et al. 1981). Passive elastic moment $\left(M_{P E}\right)$ has previously been modeled using exponential functions (Hoang et al. 2005; Riener and Edrich 1999; Yoon and Mansour 1982). Here, $M_{G}$ and $M_{P E}$ were modeled as a generalized sinusoid and the sum of two exponential terms, respectively: 


$$
M_{P E}=B_{1} e^{k_{1} \theta}+B_{2} e^{k_{2} \theta}
$$

The constants $A$ define the gravitational moment, the constants $B$ and $k$ define the passive elastic moment, and $\theta$ is a measure of joint angle in radians. Each exponential term models the passive elastic moment at one end of the ROM. Although the convention used for $\theta$ is flexible, we have adopted one such that zero represents the anatomical position, and ankle dorsiflexion, knee flexion, and hip flexion angles are positive.

Passive moment $\left(M_{P}\right)$, as measured by the dynamometer, was modeled as the sum of gravitational moment and passive elastic moment:

$$
\begin{gathered}
M_{P}=M_{G}+M_{P E}=A_{1} \sin (\theta)+A_{2} \cos (\theta) \\
+B_{1} e^{k_{1} \theta}+B_{2} e^{k_{2} \theta}
\end{gathered}
$$

All six constants in this equation must be determined to make gravitational moment corrections and to include passive elastic moments in the model.

The parameters for the passive moment model (Equation 3) were determined by fitting the model to measured passive moment data. Prior to determining model parameters, the dynamometer data were low pass filtered ( $5 \mathrm{~Hz}$ cutoff, fourth-order Butterworth filter). Because of viscoelastic effects, the measured passive moment is somewhat dependent on the direction of motion, resulting in a hysteresis loop. To reduce such an effect, the model was fit to data from a full flexion-extension cycle.

The gravitational moment constants $A_{1}$ and $A_{2}$ were first determined by fitting the gravitational moment equation (Equation 1) to the passive moment profile within the portion of the ROM where the passive elastic moments were negligible (i.e., the recorded moment was due solely to gravity). The first step in this process was defining this gravity-only region (GOR). Consider the sum of the passive moment, as defined by Equation 3, and its second derivative. The sinusoidal components of the gravitational moment cancel out, leaving only the exponential terms of the passive elastic moment:

$$
\begin{gathered}
M_{P}+\frac{d^{2} M_{P}}{d^{2} \theta}=M_{P E}+\frac{d^{2} M_{P E}}{d^{2} \theta}=B_{1}(1 \\
\left.+k_{1}^{2}\right) e^{k_{1} \theta}+B_{2}(1 \\
\left.+k_{2}^{2}\right) e^{k_{2} \theta}
\end{gathered}
$$

The second derivative of the passive elastic moment (Equation 2) may not be negligible throughout the GOR, but will pass through zero where the slope of the function is at a minimum. Thus, at some central point within the GOR Equation 4 will go to zero. Based on this, an estimate of the center of the GOR can be made based on the passive moment data. A best fit cubic polynomial of the passive moment profile was determined using a least squares approach and added to its second derivative, giving: 


$$
\begin{aligned}
M_{F I T}+ & \frac{d^{2} M_{F I T}}{d \theta^{2}} \\
& =a_{1} \theta^{3}+a_{2} \theta^{2}+\left(6 a_{1}+a_{3}\right) \theta+2 a_{2}+a_{4}
\end{aligned}
$$

The center of the GOR was defined using the real root of the cubic polynomial in Equation 5 (Figure 1).

The gravitational moment constants were then determined by a least squares fit of Equation 1 to the passive moment data in the GOR (Figure 2). The GOR was defined as a span of $40 \%$ of the joint ROM centered at the center of the GOR. This span was estimated by examining passive elastic moment functions calculated previously using an optimization approach (Anderson et al. 2007), which indicated that in general the passive elastic moment is negligible for a minimum of $40 \%$ of the ROM.

The passive elastic moment constants $B$ and $k$ were then determined from the passive moment data following gravity correction. The gravitational moment function found previously was subtracted from the passive moment profile, producing the passive elastic moment data (Figure $3)$. Then, the data were split at the center of the GOR. In each portion, the parameters $B$ and $k$ of the exponential fit were estimated using the nonlinear least-squares fitting tool (nlinfit) in Matlab (The MathWorks, Natick, Massachusetts, USA). The two portions were added together to determine the passive elastic moment function (Equation 2).

The performance of the method described here was evaluated by calculating model goodness of fit using concordance correlation coefficients $\left(\rho_{c}\right)$. The concordance correlation coefficient evaluates the agreement between predicted and measured moments, that is the variation of the data pairs from a $45^{\circ}$ line through the origin (Lin 1989). It can take a value between -1 and 1 .

The performance of the method described here was also compared to gravity correction using a simple sine or cosine function based on a single data point. The angle at which gravitational moment went to zero was assumed to be known (e.g. when the dynamometer arm was vertical). A single data point was taken from the passive moment profiles at an angle chosen such that a significant gravitational moment would be present, but also with a minimal passive elastic moment (which would introduce error into the gravity correction). The angles used corresponded to neutral or slight dorsiflexion for the ankle, $\sim 60^{\circ}$ of flexion for the knee, and $\sim 40^{\circ}$ flexion for the hip. The passive moment at these angles was averaged between the two directions of motion to cancel the hysteresis effect. The root mean square (RMS) of the error was calculated for each gravity correction method within the GOR, and the results compared using paired t-tests.

\section{Results}

The method presented here produced models (Equation 3) that fit the measured passive moment profiles well for all participants. Specifically, mean values of $\rho_{\mathrm{c}}$ were 0.996 for hip flexionextension, 0.987 for knee flexion-extension, and 0.981 for ankle plantar flexion-dorsiflexion. Typical (median) values of model coefficients are presented in Table 1.

The gravitational moment corrections calculated using simple sine or cosine functions based on a single data point had greater error within the range of the GOR than corrections using the new method presented here. For the single data point method within the GOR, the mean RMS error was $5.195 \mathrm{~N}-\mathrm{m}, 1.274 \mathrm{~N}-\mathrm{m}$ and $0.972 \mathrm{~N}$ - for the hip, knee and ankle, respectively. For the new method within the GOR, the mean RMS error was $2.451 \mathrm{~N}-\mathrm{m}, 0.908 \mathrm{~N}-\mathrm{m}$, and 0.641 
$\mathrm{N}-\mathrm{m}$ for the hip, knee and ankle, respectively. Differences in RMS between methods were statistically significant for ankle ( $\mathrm{p}<0.001)$, knee $(\mathrm{p}=0.003)$ and hip $(\mathrm{p}<0.001)$.

\section{Discussion}

An improved method is presented here for the gravity correction of dynamometer data. It has been shown to work for hip, knee and ankle testing on a Biodex System 3, but should be applicable to cases involving other joints and/or dynamometers. This method has several advantages over methods based on a single data point. First, it bases the gravity correction on data from the entire joint ROM, providing a more accurate depiction of gravitational moments throughout the ROM, the only added cost being slightly greater data collection time. Second, the gravitational moment is modeled using a generalized sinusoid. This method avoids the assumption, implicit in single-data-point methods, that the angle at which the gravitational moment goes to zero is known. It should be noted that this assumption may be particularly bad for the case of the ankle, as the shape of the foot-attachment system does not lend itself to estimating how its center of mass is oriented relative to the dynamometer axis. Third, this method accounts explicitly for the presence of passive elastic moments, which are assumed to be negligible in single-data-point methods.

In addition to gravity correction, this method provides a simple method for estimating and modeling passive elastic joint moments. These moments may, in some cases, be of intrinsic interest (e.g., they can be added to models of active joint moment to create models of total joint moment). The present modeling approach may also be of use in comparing passive elastic moments between groups, such as healthy and pathologic populations.

\section{Acknowledgments}

This work was supported in part by Grant R03 OH007321 from Centers for Disease Control and Prevention. Its contents are solely the responsibility of the authors, and do not necessarily represent the official views of the sponsor.

\section{References}

Anderson DE, Madigan ML, Nussbaum MA. Maximum voluntary joint torque as a function of joint angle and angular velocity: Model development and application to the lower limb. Journal of Biomechanics 2007;40:3105-3113. [PubMed: 17485097]

Ford WJ, Bailey SD, Babich K, Worrell TW. Effect of hip position on gravity effect torque. Med Sci Sports Exerc 1994;26:230-234. [PubMed: 8164541]

Gajdosik RL, Leslie CM, Thorne-Thomsen AA. Influence of short hamstring muscles on gravitational torque of the leg and knee extension and flexion concentric isokinetic torque. Isokinetics and Exercise Science 2001;9:85-90.

Herzog W. The relation between the resultant moments at a joint and the moments measured by an isokinetic dynamometer. Journal of Biomechanics 1988;21:5-12. [PubMed: 3339027]

Hoang PD, Gorman RB, Todd G, Gandevia SC, Herbert RD. A new method for measuring passive lengthtension properties of human gastrocnemius muscle in vivo. Journal of Biomechanics 2005;38:13331341. [PubMed: 15863118]

Keating JL, Matyas TA. Method-related variations in estimates of gravity correction values using electromechanical dynamometry: a knee extension study. J Orthop Sports Phys Ther 1996;24:142153. [PubMed: 8866273]

Kellis E, Baltzopoulos V. Gravitational moment correction in isokinetic dynamometry using anthropometric data. Med Sci Sports Exerc 1996;28:900-907. [PubMed: 8832545]

Lin LI. A concordance correlation coefficient to evaluate reproducibility. Biometrics 1989;45:255-268. [PubMed: 2720055]

Nelson SG, Duncan PW. Correction of isokinetic and isometric torque recordings for the effects of gravity. A clinical report. Physical Therapy 1983;63:674-676. [PubMed: 6844413] 
Riener R, Edrich T. Identification of passive elastic joint moments in the lower extremities. Journal of Biomechanics 1999;32:539-544. [PubMed: 10327008]

Westing SH, Seger JY. Eccentric and concentric torque-velocity characteristics, torque output comparisons, and gravity effect torque corrections for the quadriceps and hamstring muscles in females. International Journal of Sports Medicine 1989;10:175-180. [PubMed: 2777436]

Winter DA, Wells RP, Orr GW. Errors in the use of isokinetic dynamometers. European Journal of Applied Physiology and Occupational Physiology 1981;46:397-408. [PubMed: 7196329]

Yoon YS, Mansour JM. The passive elastic moment at the hip. Journal of Biomechanics 1982;15:905910. [PubMed: 7166551] 


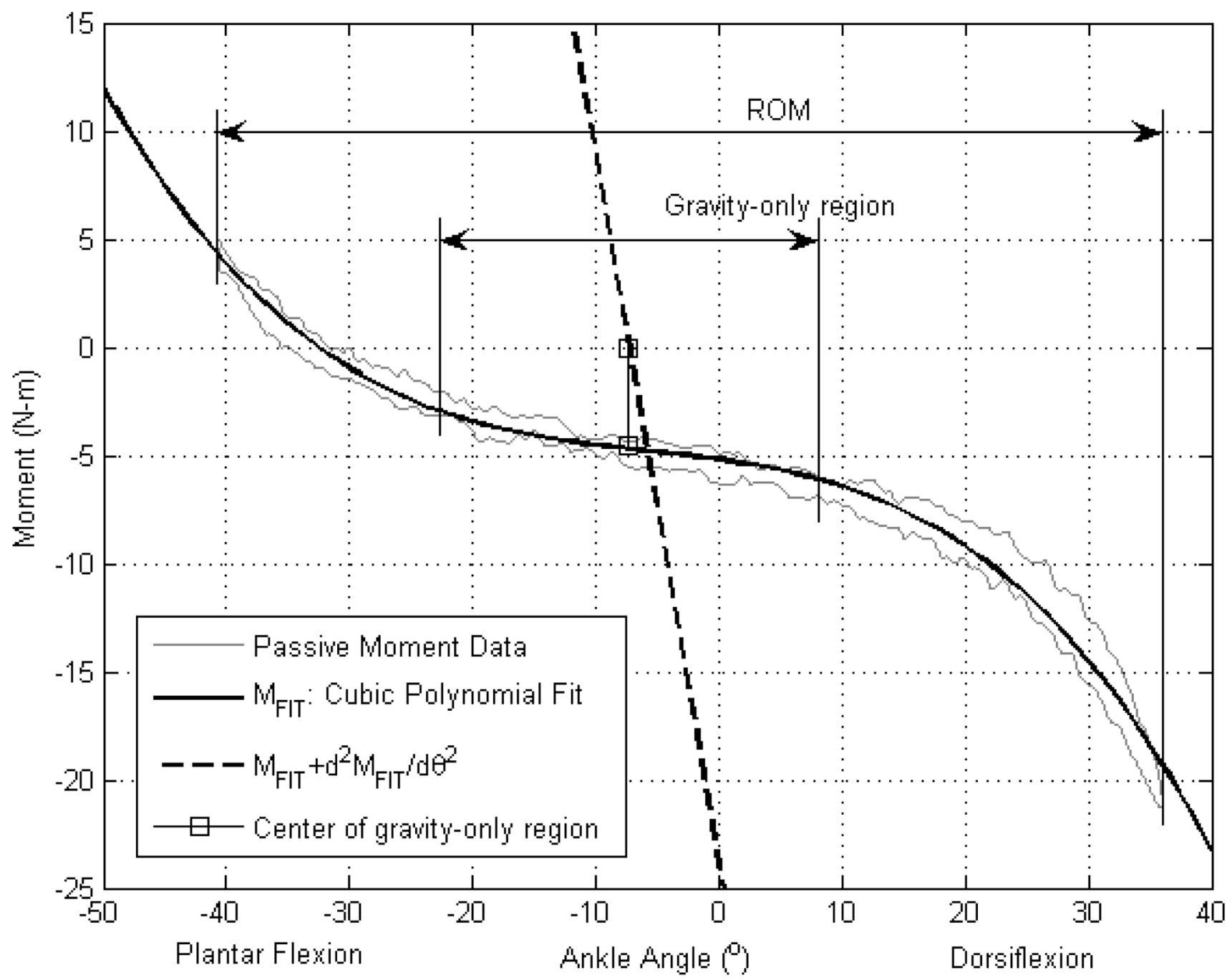

Figure 1.

Determination of the gravity-only region (GOR) of passive moment data for the ankle. The center of the GOR is defined by the root(s) of a cubic polynomial fit, $M_{F I T}$, added to its second derivative. The width of the GOR was $40 \%$ of the range of motion. 


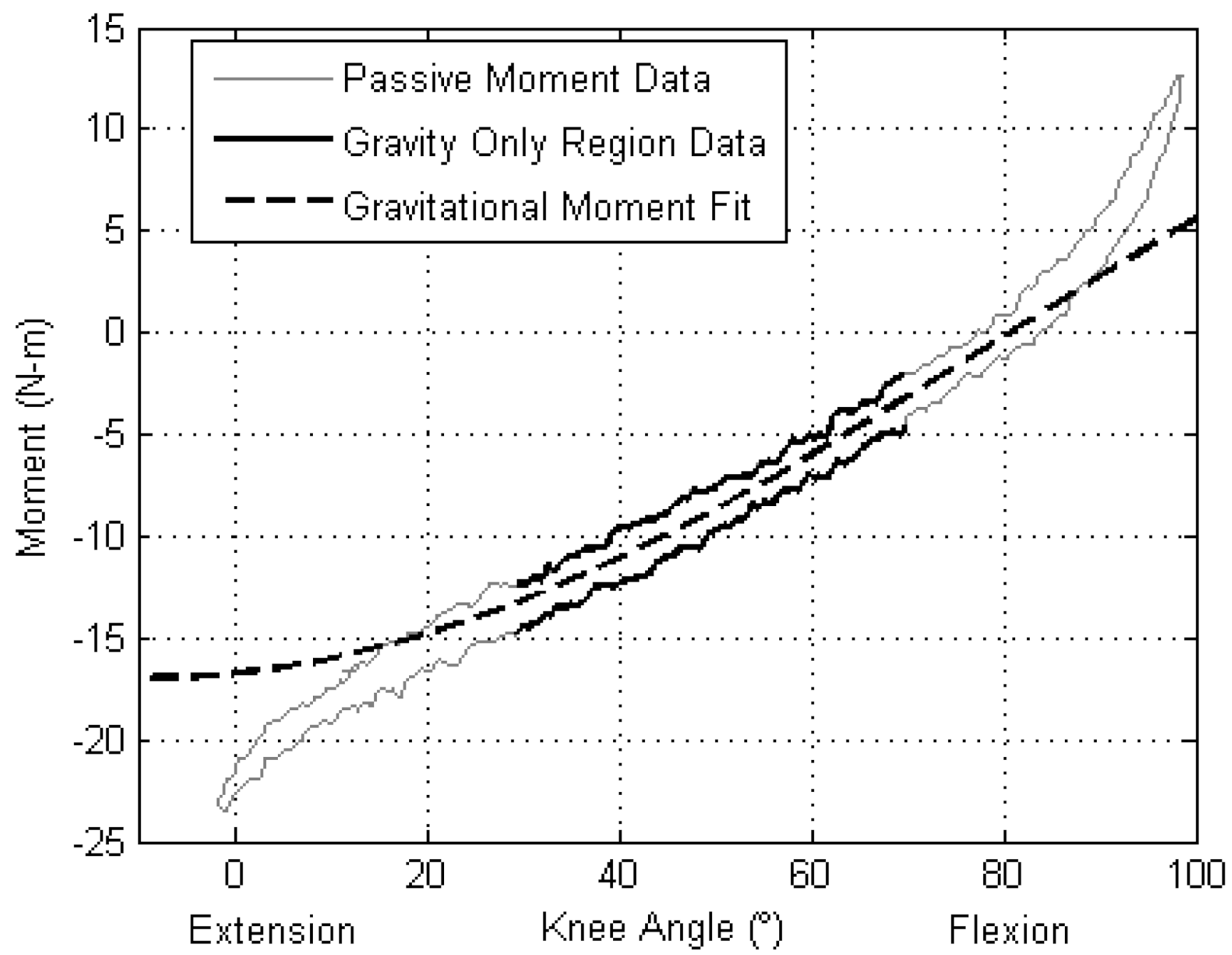

Figure 2.

Passive moment data recorded through one full cycle of knee motion (light solid line), with the estimated gravity only region (dark solid lines) and the sinusoidal gravitational moment fit (dashed line). 


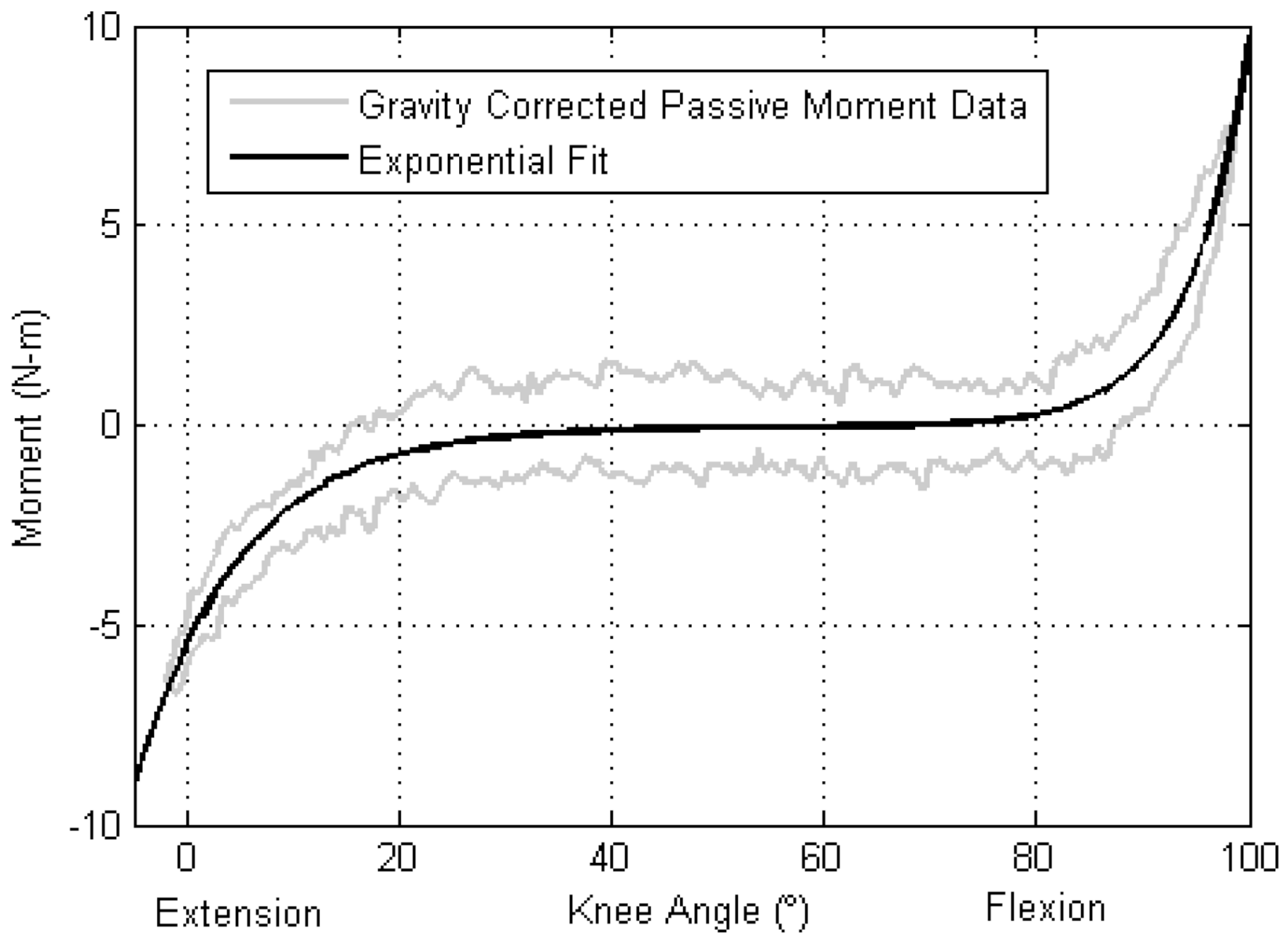

Figure 3.

Passive moment data from Figure 2 following gravity correction (light solid line). The exponential passive elastic moment model (dark solid line) is determined by nonlinear regression in each end after splitting the data in the center of the gravity only region. 


\section{Table 1}

Median passive moment model (Equation 3) coefficients determined using the method described. These models were based on joint angles, with zero representing the anatomical position, and dorsiflexion, knee flexion and hip flexion angles positive.

\begin{tabular}{|l|l|l|l|}
\hline & Ankle & Knee & Hip \\
\hline$A_{I}$ & -7.023 & 2.115 & -60.66 \\
\hline$A_{2}$ & -7.119 & -15.55 & 9.267 \\
\hline$B_{I}$ & $2.676 \mathrm{E}-5$ & $5.05 \mathrm{e}-10$ & 1.179 \\
\hline$k_{1}$ & -18.23 & 12.71 & -7.379 \\
\hline$B_{2}$ & -0.3758 & -9.572 & $-1.246 \mathrm{E}-2$ \\
\hline$k_{2}$ & 7.788 & -5.121 & 5.534 \\
\hline
\end{tabular}

